\title{
Evaluation of scientific performance of JEM-EUSO mission with Space-X Dragon option
}

\author{
K Shinozaki ${ }^{* 1}$, A Santangelo ${ }^{1}$, J Bayer ${ }^{1}$, M Bertaina $^{2}$, P Bobík ${ }^{3}$, MJ Christl ${ }^{4}$, F Fenu $^{2}$, \\ P Gorodetzky ${ }^{5}$, A Guzmán ${ }^{1}$, E Iwotschkin ${ }^{1}$, G Medina-Tanco ${ }^{6}$, T Mernik ${ }^{1}$, \\ A Neronov ${ }^{7}$, AV Olinto ${ }^{8}$, B Pastirčák ${ }^{3}$, M Putiš $^{3}$ and L Wiencke ${ }^{9}$ \\ on behalf of the JEM-EUSO Collaboration \\ ${ }^{1}$ Institut für Astronomie und Astrophysik, Universität Tübingen, Tübingen, Germany \\ ${ }^{2}$ Department of Physics, University of Torino, Turin, Italy \\ ${ }^{3}$ Institute of Experimental Physics, Slovak Academy of Science, Kosice, Slovakia \\ ${ }^{4}$ NASA/Marshall Space Flight Center, Huntsville, USA \\ ${ }^{5}$ APC, University of Paris Didetot, Paris, France \\ ${ }^{6}$ Instituto de Ciencias Nucleares, Unversidad Nacional Autónoma de México, Mexico City, \\ Mexico \\ ${ }^{7}$ ISDC Data Centre for Astrophysics, University of Geneva, Versoix, Switzerland \\ ${ }^{8}$ Department of Astronomy \& Astrophysics, Kavli Institute for Cosmological Physics, \\ The University of Chicago, Chicag, USA \\ ${ }^{9}$ Department of Physics, Colorado School of Mines, Golden, USA \\ E-mail: shinozakieastro.uni-tuebingen.de
}

Extreme Universe Space Observatory on-board the Japanese Experiment Module (JEM-EUSO) is a mission devoted to the observation of ultra-high energy cosmic rays (UHECRs) around and above the Greisen-Zatseptin-Kuzimin energy at $\sim 5 \times 10^{19} \mathrm{eV}$. The origin of these enigmatically energetic cosmic rays remain an open question since their discovery more than 50 years ago. High statistics on UHECRs are essential to provide key information to answer this question and necessitate very large exposures to overcome their extremely low flux of an order of a few events per square kilometer per century. JEM-EUSO is designed to measure the air showers induced by UHECRs using a super-wide field-of-view ultra-violet fluorescence telescope pointed downwards on Earth's nighttime atmosphere. Orbiting onboard the International Space Station (ISS), JEM-EUSO rather uniformly covers the entire Celestial Sphere, allowing a thorough analysis of the UHECR arrival direction distribution. In the present work, we introduce a design of the JEM-EUSO telescope suitable for using the Space-X Falcon 9 rocket and the Dragon spacecraft for transport to the ISS. This design allows for accommodation of the telescope with equivalent or slightly improved performance than that studied for H-II Transport Vehicle option. We then discuss the expected performance, in particular the scientific objective of searching the arrival direction distribution of UHECRs for their origin through simulation studies.

The 34th International Cosmic Ray Conference,

30 July-6 August, 2015

The Hague, The Netherlands

* Speaker. 


\section{Introduction}

The nature of the ultra-high energy cosmic rays (UHECRs), referred to as energies $E_{0} \gtrsim 5 \times$ $10^{19} \mathrm{eV}$ hereafter, remains a long standing mystery in astrophysics [1]. Their extremely low fluxes of the order of few event per $\mathrm{km}^{2}$ in centuries has limited advancement of our knowledge about them. No sources have been identified as their origin by ground-based observations even using afew-1000- $\mathrm{km}^{2}$ effective area air shower arrays. Thus, dramatic increases in observation capability are desired in the forthcoming era for UHECR physics.

The JEM-EUSO (Extreme Universe Space Observatory on-board the Japanese Experiment Module) mission [3, 4] is a novel approach to investigate UHECR [5] by fluorescence technique from space. It is designed to operate on the Exposed Facility of the JEM Kibo module of the International Space Station (ISS) [6]. At a nominal altitude $H_{0} \sim 400 \mathrm{~km}$ from Earth's surface, it completes an orbit every 90 minutes with an orbital inclination of $51.6^{\circ}$.

The main telescope of JEM-EUSO watches a large area of the nighttime atmosphere and detects the fast moving light spots resulting from air showers. Using refractive Fresnel optics [7] results in a super-wide field-of-view (FOV; $\sim 60^{\circ} \phi$ ) for observing air shower events. The focal surface (FS) is covered with an array of 137 photo-detector modules (PDMs), each of which is formed from 36 multi-anode photomultiplier tubes (MAPMTs) with 64 pixels each [8]. Utilization of silicon photomultiplier ( $\mathrm{SiPM}$ ) is also under consideration [9]. Along with elaborate electronics, the MAPMTs allow high resolution imaging with $\sim 3 \times 10^{5}$ pixel over $2.5 \mu$ s intervals. An atmospheric monitoring (AM) system [10] is used to determine the cloud coverage and properties of the atmosphere in the FOV.

In the present work, we estimate the fundamental performance of JEM-EUSO under a launch scenario utilising the Space-X Dragon spacecraft [11]. The JEM-EUSO will be mounted in the unpressurised section of the Dragon trunk for transport [12]. Space-X began regular operation in 2012 to deliver cargo to the ISS. The Dragon spacecraft is launched into orbit on Space-X's Falcon 9 rocket. Based on a preliminary design of the telescope for this option, we estimate the expected exposure for arrival direction distribution analysis and discuss the relevant issues.

\section{Observation of air showers by JEM-EUSO with Space-X Dragon option}

To simulate air shower and detector response, we employ the ESAF (EUSO Simulation and Analysis Framework) package [14]. The JEM-EUSO configuration is nealy the same described in the other scenarios [15] expect for the modifications required for accomodation in the Dragon spacecraft. The influence of clouds is modeled based on TOVS satellite database [16]. The clouds are characterised by the optical depth of the cloud; $\tau_{\mathrm{C}}$, and the height of the cloud; $H_{\mathrm{C}}$.

In Figure 1, the top panel shows the arrival time distribution of photons to JEM-EUSO from a typical air shower of $E=10^{20} \mathrm{eV}$ with a zenith angle $\Theta=60^{\circ}$ [17]. Three different atmospheric conditions are considered as described in the legend. The bottom panel displays the time-integrated images of signals on the FS detector for these cases. Signal counts per pixel are indicated by filled squares. The position along the horizontal axis corresponds to the arrival time in the top panel.

In air shower observation from space, fluorescence light is the dominant component along with a small fraction of Cherenkov light that is scattered in the atmosphere. In addition, a space-based 

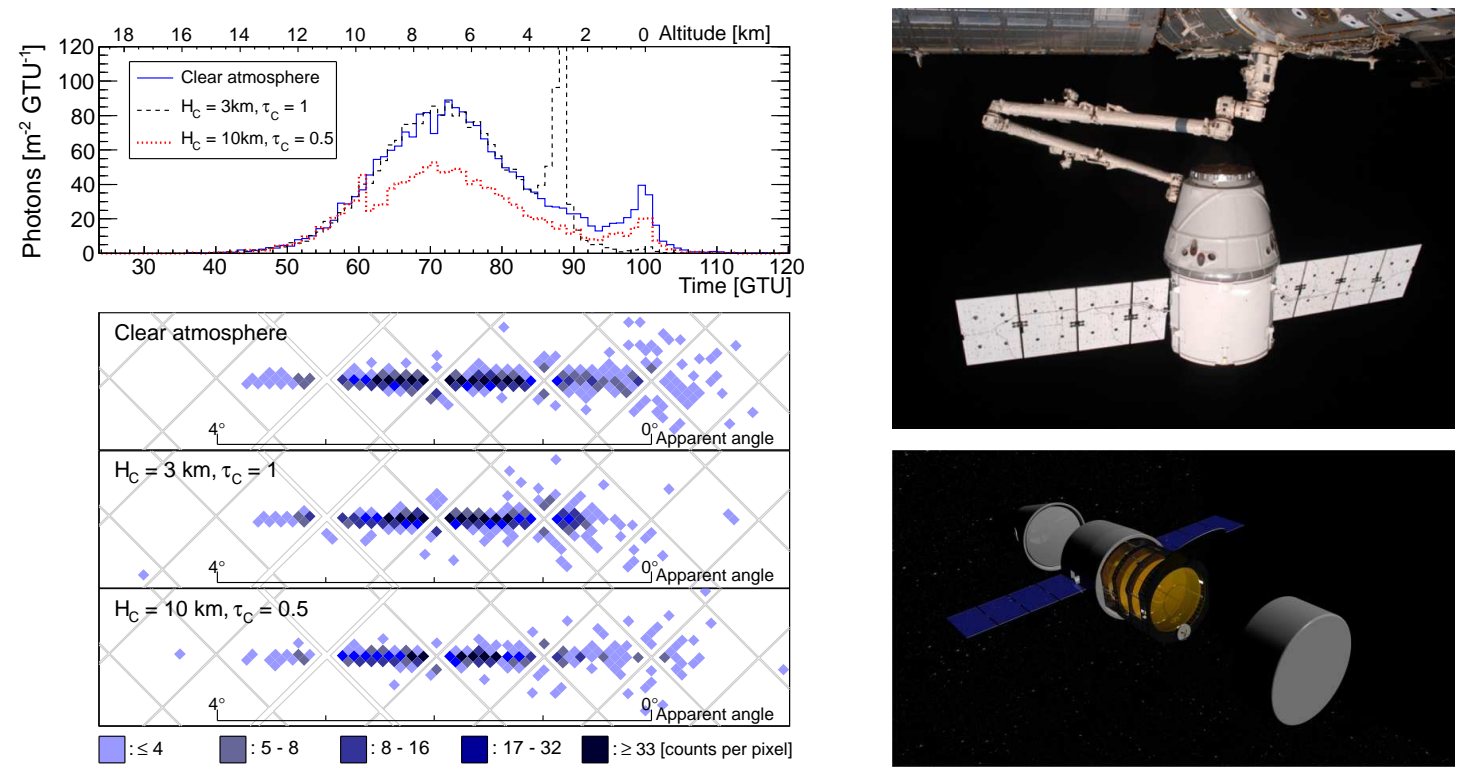

Figure 1: Arrival time distribution of photons (top panel) from a typical air shower of $E_{0}=10^{20} \mathrm{eV}$ and $\Theta=60^{\circ}$. Timeintegrated signals on the FS detector is shown (bottom) for different atmospheric conditions [17]. See text for detail.

Figure 2: Dragon trunk captured by RMS of the ISS (top panel). Artist's view of the JEM-EUSO accommodation in Dragon (bottom).

observatory may also detect the reflection of Cherenkov photons from bodies of land or water as well as clouds. These reflected signals, referred to as 'Cherenkov footprint,' can provide a valuable piece of information on the trajectory and timing of the air shower.

For air showers of a given energy, intrinsic observable properties are predominantly determined by the zenith angle. For large zenith angles, the signals incident on the detector are more intense, have a longer length of the projected track and have a longer duration. These effects all contribute to increase the efficiency of the event trigger as well as subsequent event reconstruction.

While the space-based observation technique has been investigated, the design of the JEMEUSO telescope must be modified to utilize the Dragon option. Preliminary studies have been completed using the physical dimensions of the Dragon trunk [11]. These constraints limit the dimensions of the telescope, but in general several advantages are expected in comparison to the H-II Transfer Vehicle (HTV) option.

Figure 2 shows the Dragon spacecraft captured by the remote manipulator arm (RMS) of the ISS in the top panel. An artist's view of the JEM-EUSO accommodated in Dragon is illustrated in the bottom [11].

The payload capacity of Dragon is $6000 \mathrm{~kg}$ and is shared between pressurised and unpressurised cargos. The unpressurised cargo volume in the trunk is $14 \mathrm{~m}^{3}$. The trunk is cylindrical with tapered and straight sections. The diameter of the trunk is $297 \mathrm{~cm}$. The maximum length of the payload volume in the trunk is $221 \mathrm{~cm}$. The avionics tray on the second stage of the Falcon 9 extends up into the centre of the trunk that reduces the length of the payload volume to $178 \mathrm{~cm}$. This is an annular region $46 \mathrm{~cm}$ deep lying between the avionics tray and the inner surface of the second 


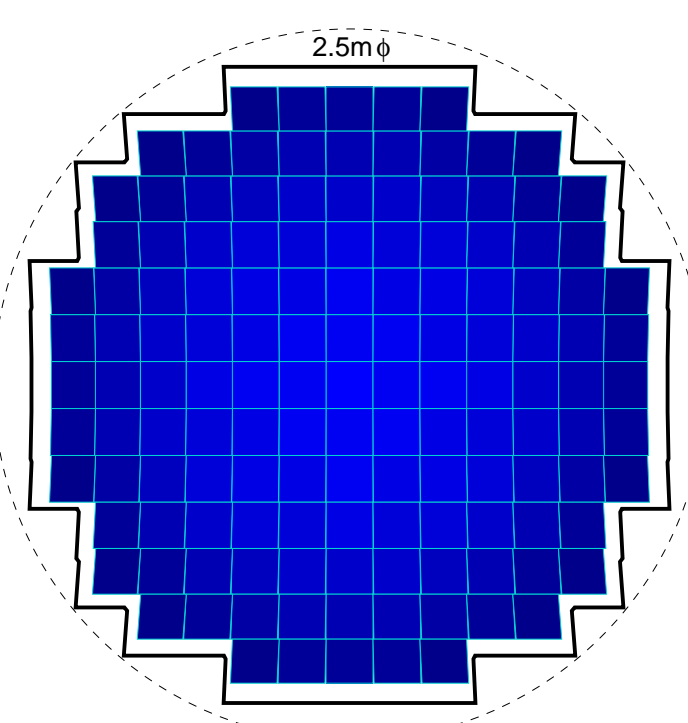

Figure 3: Alignment of 137 PDMs on the focal surface. See text for detail.

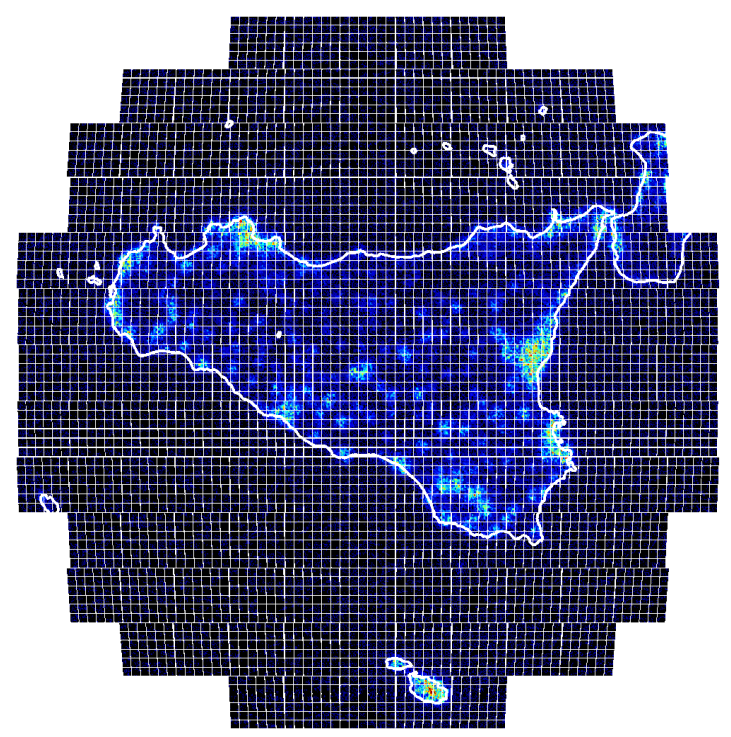

Figure 4: Illustrative image in nominal FOV seen from Caltanissetta, Sicily at $400 \mathrm{~km}$ altitude. See text for detail.

stage extension. To accommodate JEM-EUSO in the Dragon trunk several modifications to the JEM-EUSO design is investigated (see Reference [12] further details).

Figure 3 shows the alignment of 137 PDMs on the FS structure assumed in the present work. The solid line indicates the minimal area that is needed for PDMs allocation on the FS. The dashed circle represents the projection of the front lens entrance aperture and is $2.5 \mathrm{~m}$ diameter. Note that the geometry of the FS is approximately spherical and the inclination of PDM front surfaces vary up to $\sim 22^{\circ}$.

Figure 4 illustrates nominal FOV of JEM-EUSO when the ISS is flying above Caltanissetta at $H_{0}=400 \mathrm{~km}$. The image of terrestial light on Sicily, area is ca. $26,000 \mathrm{~km}^{2}$, as seen by the FS detector was generated using DMSP data [13].

In the original design for the HTV option, the JEM-EUSO telescope is attached to the HTV's Exposed Pallet at the entrance aperture of the telescope. Flying on Dragon makes it possible to attach JEM-EUSO inside the trunk by the FS structure. This should result in a design for JEMEUSO that saves weight. In the HTV design it is necessary to trim the sides of the lenses and the FS to fit in the HTV payload volume. As the Dragon trunk has a nearly circular cross section, the lenses and FS in JEM-EUSO can be round; potentially improving the aperture of the optics compared to the HTV option. By accommodating the same number of PDMs on the FS, a similar observation area $S_{\mathrm{obs}}\left[\mathrm{km}^{2}\right] \sim 1.4 \times 10^{5} \cdot\left(H_{0} / 400[\mathrm{~km}]\right)^{2}$ is achieved for nadir observation.

\section{Exposure for arrival direction distribution analysis}

For scientific objectives, the observed air shower events should be selected wtih criteria to maximise statistics and insure the quality of event reconstructions. The former is the key point of great importance that should be pursued with space-based UHECR observations. The latter is 


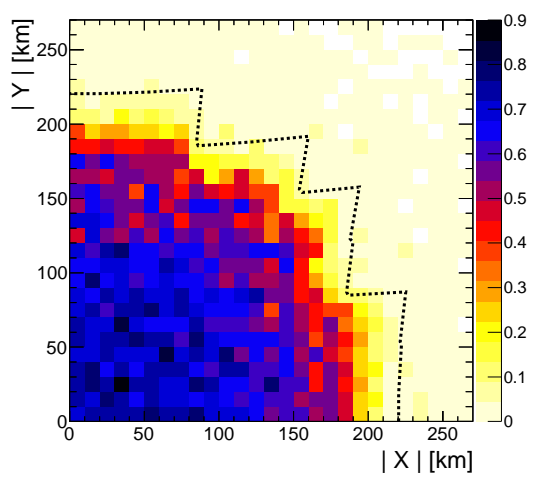

Figure 5: Probability of trigger as a Figure 6: Expected annual exposure for different criteria function of core locations for UHECRs with as a function of energy. Referential $\pm 20 \%$-uncertainty in $E_{0}>5 \times 10^{19}[\mathrm{eV}]$. See text for details.

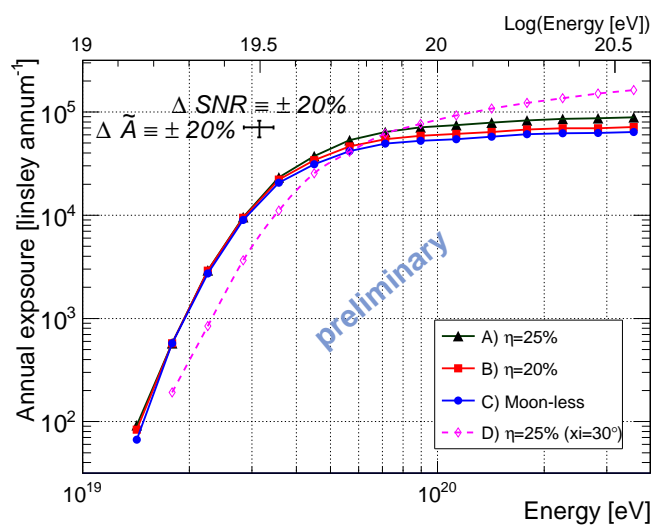

SNR and $\tilde{A}$ are indicated. See text for detail.

less pronounced compared to the ground-based observatories, even though proper selections are needed to achieve the scientific object. In the present section, we discuss the expected exposure for the different event selection criteria. To compare with other experiments, 'annual exposure' is used, which gives the average growth of exposure for each year of operation. To estimate it, we first evaluate the instantaneous aperture $a_{\text {sel }}$ for given criteria by generating a large amount of simulated air showers. The trigger algorithms at two sequential levels are then applied to this data $[18,19]$.

In Figure 5, the trigger probability as a function of core location within the FOV is shown by colour scale for incident UHECRs with $E_{0}>5 \times 10^{19} \mathrm{eV}$. The coordinate of the core locations is given by each axis. Fluxes of UHECRs observed by the Pierre Auger Observatory [20] are assumed to weight each simulated event. Dashed lines indicate the boundary of the nominal FOV viewed for the outer most MAPMTs.

All the data from the FS detector for triggered events are then analysed. Among simulated air showers with energy $E_{0}$ in the area $S_{\text {sim }} \gg S_{\text {obs }}$ and solid angle acceptance $\Omega_{\text {sim }}=\pi$ [sr], for any arrival direction with $\Theta=0^{\circ}$ to $90^{\circ}$, the selection probability $P_{\text {sel }}$ is evaluated; $a_{\text {sel }}\left(E_{0}\right) \equiv S_{\text {sim }} \Omega_{\text {sim }}$. $P_{\text {sel }}\left(E_{0}\right)$. In the present work, the reference $a\left(E_{0}\right)$ is calculated under a constant background level. The dominant background source is airglow, while moonlight, can also contribute via reflection from Earth's atmosphere or surface. Recent EUSO-Balloon measurements have shown a positive correlation between the background intensity and cloud presence [21].

These factors may be empirically scaled by the signal-to-noise ratio (SNR) with respect to the reference $a\left(E_{0}\right)$. Approximately, the SNR is linearly increased by the overall efficiency of photon detection and is reduced by square root of background level [15]. The former is applicable to using different FS detectors such as SiPM. The latter includes the effect of clouds which is preliminarily assumed by model [14]. The effect of moonlight and the fraction of areas with intense local light $\left(f_{\text {loc }}=10 \%\right)$ have been studied [15]. Including these factor one can estimate the exposure for given selection criteria as follows:

$$
\tilde{A}\left(E_{0}\right)=\int_{0}^{T_{\mathrm{opr}}} a_{\mathrm{sel}}\left(\mathrm{SNR} \cdot E_{0}\right) d t \cdot\left(1-f_{\mathrm{loc}}\right)
$$


The SNR is also a function of time due to variability in the background level during the operation period $T_{\mathrm{opr}}$.

In Figure 6, expected annual exposures are plotted for different selection criteria as a function of energy in units of $\mathrm{km}^{2} \mathrm{sr} \mathrm{yr}$ (=linsley) per annum. Closed triangles and circles represent different observational duty cycle $\eta$ of: A) $25 \%$ and B) 20\%, respectively. Closed squares denote: C) moonless condition for $\eta=17 \%$. Open diamonds show: D) estimated values for the case of A) but tilting JEM-EUSO $30^{\circ}$ from the nadir. Uncertainty of $\pm 20 \%$ in SNR and exposure are shown as error bars as a reference.

The fraction of time that the ISS stays in the Earth's umbra is $\sim 34 \%$, otherwise SNR $=0$ is applied to Equation (3.1) due to sunlight. Allowing higher background level increases the observational duty cycle; the fraction of time that air shower observations can be made. The JEMEUSO trigger algorithms apply a dynamical threshold setting that depends on the background level provided it is not too high to prevents operating properly in photon counting mode. It should be noted that they are not restricted simply by the presence of the moon in the sky. The case A) is still below this limit, while B) has been the case assumed in our previous publications [15, 17].

Curve D) shows the estimated annual exposure for tilting JEM-EUSO by adjusting the observation area and SNR relative to those for the nadir observation [22]. $S_{\text {obs }}$ approximately scales by $\propto 1 / \cos ^{3} \xi$ where $\xi$ is the angle of tilting JEM-EUSO from the nadir. This tilting helps compensate for a decrease of observation area in case the ISS orbits at lower altitudes. Compared to the nadir observation, the distance to air showers from JEM-EUSO increases by a factor of $\sim 1 / \cos \xi$. The off-nadir observations have the potential to increase exposure at the highest energies where UHECR flux is extremely low.

In Figure 7, the occurrence of clear atmosphere (CA) or low-cloud (LC) is shown in the region where the ISS orbits assuming the TOVS data [16] (quoted from Reference [17]). The criteria of CA and LC are $\tau_{\mathrm{C}}<0.1$ and $H_{\mathrm{C}}<3.2 \mathrm{~km}$, respectively. These conditions allow measurement of the shower development for better event reconstruction (see also Figure 1).

Even in the case with low-cloud, the so-called shower maximum is observable above the cloud in most cases except for air showers at small zenith angles [15, 17]. Determinations of $X_{\max }$, the atmospheric depth at shower maximum and energy are feasible with reasonable quality [23].

Determination of the arrival direction [24] is a stable part of the data analysis. Since it only depends on the apparent velocity of light spot movement along the shower axis, observation of shower maxima is not essential despite a reduced trigger probability for cloudy conditions. In addition to the favourable conditions assumed in Figure 7, most triggered air shower events contribute to the statistics for arrival direction distribution analysis [25]. In particular at the highest energies, the number of events can increase by analysing the events obtained in higher background and/or cloudy conditions. A recent study indicates that the errors in arrival direction are mainly correlated to the intensity of the signals on the FS detector [25], independent of atmospheric conditions.

In Figure 8, the uniformity of exposure is shown in equatorial coordinates for the case A) in Figure 6 at the highest energies. The colour scale corresponds to the deviation that ranges within $\sim \pm 10 \%$ from the average over the entire Celestial Sphere.

It is worthwhile to note that unlike ground-based observatories JEM-EUSO has better sensitivity for air showers with large zenith angles. Observation of the entire Celestial Sphere is feasible thanks to the orbitting platform of the ISS. After a few years of operation, a high degree of unifor- 


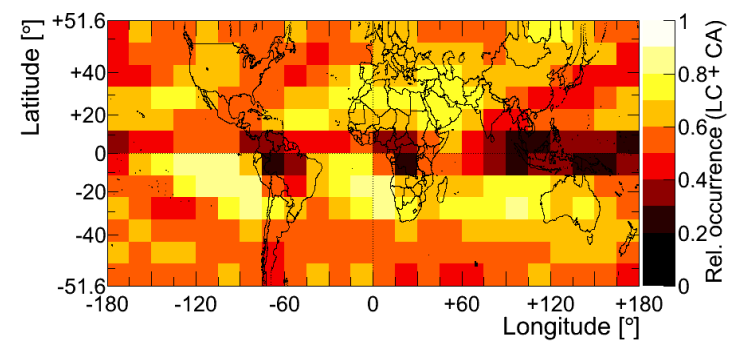

Figure 7: Occurrence of clear atmosphere or lowcloud on geographical coordinates, based on the TOVS database [16]. See text for details.

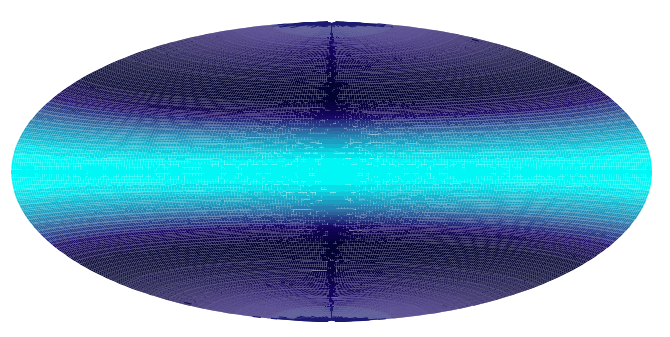

Figure 8: Exposure uniformity on equatorial coordinates for Case A) in Figure 6. The range colour scale corresponds to $\sim \pm 10 \%$ deviation.

mity in exposure will be achieved. Minor inhomogeneity appears periodically in right ascension related to the moon phase as well as seasonal variation of twilight-free observation time. The cloud effect depends on energy and tends to affect uniformity along declination according to latitudinal dependence seen in Figure 7.

\section{Summary and discussion}

In the present work, we discuss the expected performance of JEM-EUSO adopting the Space$\mathrm{X}$ Dragon option. Based on the current design study for the accommodation of the JEM-EUSO in the Dragon trunk, the expected exposures are estimated through simulation studies. The status of this option can be found in Refernce [4].

The preliminary result indicates that above $\sim 6 \times 10^{19} \mathrm{eV}$ the expected annual exposure is $\sim(5-9) \times 10^{4}$ linsleys per annum, depending on the observational duty cycle applied to data acquisition. This corresponds to about $6-10$ times higher than can be potentially achieved by Auger. Thanks to the orbiting platform of the ISS, full celestial sky coverage of UHECRs is achievable with a high degree of uniformity in a few years of operation.

These features are considerable advantages compared to the ground-based observatories. In particular for the arrival direction distribution analysis, anisotropy studies with high statistics will be feasible with JEM-EUSO without geographical constraints. So far, both Auger and Telescope Array reported at least $\sim 3 \sigma$-level significant excesses in arrival direction distribution separately observed in each hemisphere. Furthermore, a discrepancy in energy scales of UHECR energy spectra has been extensively argued. Observations with a single detector like JEM-EUSO allows reducing such systematic effects in the reconstruction of observed air showers and for example, helps clarify the reason of these results of UHECR source distribution, unifies all events to a single energy scale and may clarify any further differences reported from experiments with restricted observation capabilities.

Acknowledgment: The present work was partially supported by Basic Science Interdisciplinary Research Projects of RIKEN and JSPS KAKENHI Grant (22340063, 23340081, and 24244042), by the Italian Ministry of Foreign Affairs and International Cooperation, by the 'Helmholtz Alliance for Astroparticle Physics HAP' funded by the Initiative and Networking Fund of the Helmholtz Association, Germany, partially supported by PAPIIT-UNAM/CONACyT, Mexico and by Slovak Academy of Sciences 
MVTS JEM-EUSO as well as VEGA grant agency project 2/0076/13. Russia is supported by the Russian Foundation for Basic Research Grant No 13-02-12175-ofi-m. The Spanish Consortium involved in the JEM-EUSO Space Mission is funded by MICINN \& MINECO under the Space Program projects: AYA2009-06037-E/AYA, AYA-ESP2010-19082, AYA-ESP2011-29489-C03, AYA-ESP2012-39115-C03, AYA-ESP2013-47816 - C4, MINECO / FEDER-UNAH13-4E-2741, CSD2009-00064 (Consolider MULTIDARK) and by Comunidad de Madrid (CAM) under projects S2009/ESP-1496 \& S2013/ICE-2822. KS wishes to thank ISDC Data Centre for Astrophysics, University of Geneva where the key part of the present work was carried out for its kind support and hospitality during his stay. The computational facilities of Institute of Experimental Physics, SAS Kosice, of RIKEN Cluster of Clusters (RICC) and of RIKEN Hokusaki Great Wave System have been utilized for simulations.

\section{References}

[1] For review, A Letessier-Selvon and T Stanev, Rev. Mod. Phys. 83, 907 (2011).

[2] T Abu-Zayyad et al. (Telescope Array and Pierre Auger Collaborations), Proc. of 33rd ICRC (2013); arxiv/1310.0647.

[3] Y Takahashi et al., New J. Phys. 11, 065009/1-21 (2009);

[4] P Picozza et al., in these proceedings, \#0694 (2015).

[5] AV Olinto et al. (JEM-EUSO Collaboration), in these proceedings, \#0735 (2015).

[6] NASA, ISS User's Guide-Release 2.0 (2000); JAXA Human Space Systems and Utilization Program Group, Kibo Handbook (2007).

[7] Y Takizawa et al. (JEM-EUSO Collaboration), Proc. of 32nd ICRC (Rio De Janerio), \#832 (2013).

[8] F Kajino et al. (JEM-EUSO Collaboration), Proc. of 32nd ICRC (Rio de Janeiro), \#1128 (2013).

[9] A Haungs et al. (JEM-EUSO Collaboration), in these proceedings, \#0897 (2015).

[10] A Neronov et al., Proc. of 33rd ICRC (Beijing), \#1072 (2013).

[11] http://www.spacex.com

[12] J Adams et al. (JEM-EUSO Collaboration), Proc. of 33rd ICRC (Rio De Janeiro), \#1256 (2013).

[13] http://www.ngdc.noaa.gov/dmsp/

[14] C Berat et al., Astropart. Phys. 33, 211 (2010).

[15] JH Adams Jr. et al.Astropart. Phys. 44, 76 (2013).

[16] TOVS (TIROS Operational Vertical Sounder), http://www.ozonelayer.noaa.gov/action/tovs.htm/

[17] JEM-EUSO Collaboration, Experi. Astron., doi: 10.1007/s10686-014-9377-2 (in press; 2014).

[18] O Catalano et al. (JEM-EUSO Collaboration), Proc. 31st ICRC (Lodz), \#0326 (2009).

[19] J Bayer et al. (JEM-EUSO Collaboration), Proc. of 33rd ICRC (Rio de Janeriro), \#432 (2013).

[20] Estimated from www.auger.org based on F Salamida et al., Proc. 32nd ICRC (Beijing), 2, 145 (2011).

[21] S Mackovjak et al. (JEM-EUSO Collaboration), in these proceedings, \#1302 (2015).

[22] JEM-EUSO Collaboration, Experi. Astron., 10.1007/s10686-014-9376-3 (in press; 2014).

[23] F Fenu et al. (JEM-EUSO Collaboration), in these proceedings, \#0661 (2015).

[24] A Guzmán et al. (JEM-EUSO Collaboration), in these proceedings, \#0570 (2015).

[25] JEM-EUSO Collaboration, (in preparation). 